\title{
CONCERNING A NEW TRANSCENDENT, ITS TABULATION AND APPLICATION IN ANTENNA THEORY*
}

\author{
BY \\ C. J. BOUWKAMP \\ Natuurkundig Laboratorium der N. V. Philips' Gloeilampenfabrieken, Eindhoven
}

1. Introduction. As is well known, the integral sine and cosine functions $\operatorname{Si}(z)$ and $C i(z)$, respectively, are frequently met with in problems of applied mathematics. As an example we may mention the theory of antenna radiation, though in this field one preferably uses a slightly different pair of functions $S(z), C(z)$ defined by

$$
E(z)=C(z)+i S(z)=\int_{0}^{z}\left(1-e^{-i t}\right) d t / t,
$$

wherein $i$ denotes the imaginary unit. Obviously $S(z)$ is identical to $S i(z)$ :

$$
S(z)=S i(z)=\int_{0}^{z} \sin t d t / t .
$$

Further, ${ }^{1}$ if $\gamma$ denotes Euler's constant,

$$
C(z)=\gamma+\log z-C i(z)=\int_{0}^{z}(1-\cos t) d t / t .
$$

Recently, the author was led to the study of another transcendental function closely related to that defined by (1). This new function $E_{1}(z)$ is related to $E(z)$ in the same way as the latter is to the ordinary trigonometric functions, viz.

$$
E_{1}(z)=\int_{0}^{z} E(t) d t / t=\int_{0}^{1} \int_{0}^{1}\left(1-e^{-i z s t}\right) d s d t / s t .
$$

The function $E_{1}(z)$ was encountered in antenna theory but may possibly be of some value in other fields as well. Therefore it is thought worth while to treat some of its features here. In addition, a short table of numerical values may be of general interest. Finally, the function $\alpha_{2}(x)$, as it occurs in Hallén's antenna theory, is shown to be expressible in terms of the functions $E(x)$ and $E_{1}(x)$.

2. Power series and asymptotic expansion for $E_{1}(z)$. With respect to numerical evaluation, especially for small values of $z$, a power-series development may serve the purpose. After expanding the integrand in (1) into powers of $t$, one simple integration leads to a power series for $E(z)$. Using the latter in the left-hand integral of (4) we obtain, after another term-by-term integıation, the required expansion immediately, viz.

$$
E_{1}(z)=-\sum_{n=1}^{\infty} \frac{(-i z)^{n}}{n^{2} \cdot n !} .
$$

* Received Nov. 26, 1946.

${ }^{1}$ In our opinion, the short notation $C(z)$ for the integral (3) is to be preferred to those like $\overline{C i}(z)$ or $\operatorname{Cin}(z)$, as suggested by some authors. Then $E(z)$ may be a suitable abbreviation for the combination (1) analogous to the familiar $\exp (i z)=\cos z+i \sin z$. 
For large values of $z$, however, an asymptotic expansion is more desirable. In this respect we have found the following development:

$$
E_{1}(z) \sim A+B \log z+(\log z)^{2} / 2+\frac{e^{-i z}}{i z} \sum_{n=1}^{\infty} n !\left(1+\frac{1}{2}+\frac{1}{3}+\cdots+\frac{1}{n}\right)(i / z)^{n},
$$

where the constants $A$ and $B$ are given by

$$
\begin{aligned}
& A=\gamma^{2} / 2-\pi^{2} / 24+\pi \gamma i / 2=-0.2446445548+0.9066845943 i, \\
& B=\gamma+\pi i / 2=0.5772156649+1.5707963268 i .
\end{aligned}
$$

Formula (6) may be proved as follows: An equivalent definition of $E_{1}(z)$ is

$$
E_{1}(z)=-\int_{0}^{1}\left(1-e^{-i z t}\right) \log t d t / t
$$

as can be verified by a partial integration of the left-hand integral in (4), and an obvious change in the variable of integration. Once more integrating by parts one is led to

$$
E_{1}(z)=\frac{1}{2} i z \int_{0}^{1} e^{-i z t}(\log t)^{2} d t .
$$

Now, for large $e^{2}$ values of $z$, the main contribution to the integral (8) comes from the values of the integrand in the neighbourhood of $t=0$. It is therefore reasonable to consider the integral (8) as the sum of two terms:

$$
E_{1}(z)=H(z)+h(z)
$$

where the "main term" and the "correction term" are defined by

$$
\begin{aligned}
H(z) & =\frac{1}{2} i z \int_{0}^{-i \infty} e^{-i z t}(\log t)^{2} d t, \\
h(z) & =\frac{1}{2} i z \int_{-i \infty}^{1} e^{-i z t}(\log t)^{2} d t,
\end{aligned}
$$

respectively.

Let us first transform the main term $H(z)$. Evidently one can transform the expression (10) into

$$
H(z)=\frac{1}{2} i z\left[\frac{d^{2}}{d s^{2}} \int_{0}^{-i \infty} t^{s-1} e^{-i z t} d t\right]_{s=1} \cdot
$$

Now, from gamma-function theory, we have

$$
\int_{0}^{-i \infty} t^{-s-1} e^{-i z t} d t=\Gamma(s) /(i z)^{s} .
$$

Consequently, upon performing the differentiations,

$$
H(z)=\Gamma^{\prime \prime}(1) / 2-(\log z+\pi i / 2) \Gamma^{\prime}(1)+(\log z+\pi i / 2)^{2} \Gamma(1) / 2 .
$$

\footnotetext{
${ }^{2}$ Henceforth we suppose $z>0$.
} 
Finally, after substitution of the known numerical constants

$$
\Gamma(1)=1, \quad \Gamma^{\prime}(1)=-\gamma, \quad \Gamma^{\prime \prime}(1)=\gamma^{2}+\pi^{2} / 6,
$$

one easily finds

$$
H(z)=A+B \log z+(\log z)^{2} / 2,
$$

wherein the coefficients $A, B$ are as specified above.

Concerning the correction term $h(z)$ we proceed as follows: Let

$$
g(t)=(\log t)^{2} / 2
$$

then, by successive partial integrations of (11),

$$
h(z)=e^{-i z}\left[g(1)+\frac{g^{\prime}(1)}{i z}+\cdots+\frac{g^{(n)}(1)}{(i z)^{n}}\right]-\frac{1}{(i z)^{n}} \int_{1}^{-i \infty} g^{(n+1)}(t) e^{-i z t} d t .
$$

Further, by induction, or otherwise,

$$
\begin{gathered}
g^{(n+1)}(t)=(-1)^{n} n ! t^{-n-1}\left(\log t-1-\frac{1}{2}-\cdots-\frac{1}{n}\right), \\
g(1)=g^{\prime}(1)=0, \quad g^{(n+1)}(1)=(-1)^{n+1} n !\left(1+\frac{1}{2}+\frac{1}{3}+\cdots+\frac{1}{n}\right) .
\end{gathered}
$$

We thus obtain for $h(z)$, after $N=n-1$ terms,

$$
h(z)=\frac{e^{-i z}}{i z} \sum_{n=1}^{N} n !\left(1+\frac{1}{2}+\frac{1}{3}+\cdots+\frac{1}{n}\right)(i / z)^{n}+R_{N}(z),
$$

where the remainder $R_{N}(z)$ is given by

$$
R_{N}(z)=\frac{i^{N+1}(N+1) !}{z^{N+1}} \int_{1}^{-i \infty} \frac{e^{-i z t}}{t^{N+2}}\left\{1+\frac{1}{2}+\cdots+\frac{1}{N+1}-\log t\right\} d t
$$

Moreover, it can be shown that

$$
\begin{aligned}
& \left|R_{N}(z)\right| \leqq\left(1+\frac{2 /(N+1)}{1+1 / 2+\cdots+1 /(N+1)}\right) \times \mid \text { last term taken into account } \mid, \\
& \left|R_{N}(z)\right| \leqq\left(1+\frac{1 / z}{1+1 / 2+\cdots+1 /(N+1)}\right) \times \mid \text { first term not taken into account } \mid .
\end{aligned}
$$

Further details are left to the reader.

We have thus proved the validity of the asymptotic expansion (6) for positive values of $z$, though (6) holds for $\operatorname{Re}(z)>0$ as well.

3. A third development for the function $E_{1}(z)$. For moderate values of $z$ (for instance $z=10$ ) neither the power series (5) nor the asymptotic development (6) is very useful for numerical purposes, as then too many terms are required. For such values of $z$ it is better to apply the Taylor series.

To that end we use the obvious formula

$$
E_{1}(z+\Delta)=E_{1}(z)+\sum_{n=0}^{\infty} \frac{d^{n}}{d z^{n}}\left\{\frac{E(z)}{z}\right\} \frac{\Delta^{n+1}}{(n+1) !} \equiv E_{1}(z)+\sum_{n=1}^{\infty} \frac{\Delta^{n}}{n !} c_{n}(z) .
$$


The series (13) converges for all values of $\Delta$ because $E_{1}(z+\Delta)$ is an integral funct:on of $\Delta$. The following recurrence relations for the coefficients $c_{n}(z),(n>0)$, can be established:

$$
z^{2} c_{n+2}(z)+(2 n+1) z c_{n+1}(z)+n^{2} c_{n}(z)=(-1)^{n+1} i^{n} e^{-i z},
$$

with initial values

$$
c_{1}(z)=E(z) / z, \quad c_{2}(z)=\left[1-e^{-i z}-E(z)\right] / z^{2} .
$$

Given $z$, the functions $c_{n}(z)$ can be calculated success ively. As at present there exist very accurate tables for the integral sine and cosine functions, ${ }^{3}$ it is not difficult to prepare an auxiliary table for the function $E(z)$.

4. A short table for the function $E_{1}(z)$. We have prepared a short six-decimal table for $E_{1}(z)$ for values of $z$ between 0.0 and 20.0 at intervals of length 0.2 . For $z \leqq 5.0$ the power series was applied, up to 8 decimals. For $5.0 \leqq z \leqq 20.0$ the function was computed by means of (13), the functions $c_{n}(z)$ being pre-calculated ( 8 decimals) for $z=5,7, \cdots, 19$. Accordingly, (13) was successively applied with values of $|\Delta|$ not exceeding $\mathbf{1 . 0}$.

The value of $E_{1}(20)$, obtained in this way, was checked by application of the asymptotic series for $z=20$. The difference appeared only two units of the eighth decimal. The values of $E_{1}(10)$ and $E_{1}(15)$ were also checked, by comparison with the power-series values. Moreover the eight-decimal numbers on the worksheet were checked by calculating sixth-order differences, and then rounded off to six decimals. Therefore, it will be very unlikely that the error therein exceeds half a unit of the last decimal.

Tables I, II contain the real and imaginary parts of the function $E_{1}(z)$, respectively. Thus they give

$$
\begin{aligned}
& \operatorname{Re} E_{1}(z)=\int_{0}^{z}[\gamma+\log t-C i(t)] d t / t=z \int_{0}^{1} \sin z t(\log t)^{2} d t / 2, \\
& \operatorname{Im} E_{1}(z)=\int_{0}^{2} S i(t) d t / t=z \int_{0}^{1} \cos z t(\log t)^{2} d t / 2 .
\end{aligned}
$$

5. Hallén's second-order function $\alpha_{2}(x)$. Hallén ${ }^{4}$ derived the following expression for the self-impedance of the center-fed perfectly conducting cylindrical antenna

$$
Z(x)=-60 i \Omega \frac{\cos x+\alpha_{1}(x) / \Omega+\alpha_{2}(x) / \Omega^{2}+\cdots}{\sin x+\beta_{1}(x) / \Omega+\beta_{2}(x) / \Omega^{2}+\cdots} .
$$

In this formula $\Omega$ denotes a large constant, depending on the radius $a$ and the halflength $l$ of the antenna: $\Omega=2 \log (2 l / a)$. Further $x=k l=2 \pi l / \lambda$, where $\lambda$ is the wavelength.

Only the first-order coefficients $\alpha_{1}(x)$ and $\beta_{1}(x)$ can be given explicitly in terms of known functions, namely

$$
\begin{aligned}
& \alpha_{1}(x)=\frac{1}{2} e^{i x} E(4 x)-\cos x E(2 x) . \\
& \beta_{1}(x)=\frac{1}{2} i e^{i x}\{E(4 x)-4 E(2 x)\}+\sin x\{\log 4-E(2 x)\} .
\end{aligned}
$$

${ }^{8}$ Tables of sine, cosine and exponential integrals, vols. I, II; New York (1940). Table of sine and cosine integrals; New York (1942). (Federal Works Agency, W.P.A., City of New York. )

${ }^{4}$ Erik Hallén, Nova acta reg. soc. sci. Upsaliensis (4) 11, 1044 (1938). 
Rather intricate formulae were given for the second-order coefficients $\alpha_{2}(x)$ and $\beta_{2}(x)$, which were evaluated by graphical methods. ${ }^{5}$ It may be noticed that in the refined theory ${ }^{6}$ the same second-order coefficients occur.

Recently $\alpha_{2}(x)$ was found to be expressible in terms of $E(x)$ and $E_{1}(x)$ by means of a fairly simple formula, viz.

$$
\begin{aligned}
\alpha_{2}(x)= & -\alpha_{1}(x)\{\log 4+E(2 x)\}-\cos x E^{2}(2 x) / 2 \\
& +2 i \sin x E_{1}(4 x)+\cos x\left\{E_{1}(4 x)-2 E_{1}(2 x)\right\} .
\end{aligned}
$$

With the help of our tables for the function $E_{1}(x)$, and the American tables for Si $(x), C i(x)$, we have calculated $\alpha_{1}$ and $\alpha_{2}$ to six decimals for $0.0(0.1) 5.0$. After careful checking, these results were rounded off to four decimals. The final data are given in tables III, IV, whereby

$$
\alpha_{1}(x)=\alpha_{1}^{\mathrm{I}}(x)+i \alpha_{1}^{\mathrm{II}}(x), \quad \alpha_{2}(x)=\alpha_{2}^{\mathrm{I}}(x)+i \alpha_{2}^{\mathrm{II}}(x) .
$$

Comparison of our table for the first-order coefficient $\alpha_{1}(x)$ with those of King and Blake ${ }^{7}$ shows only small differences in the last decimal. Also the values of the second-order coefficient $\alpha_{2}(x)$ are in good agreement with the corresponding twodecimal values obtained by graphical integration. ${ }^{5}$

As for the other second-order coefficient, we do not think it possible to express $\beta_{2}(x)$ in such a simple way; unfortunately, more intricate functions seem to play a part.

In the following sections a proof of formula (17) will be given. As a rather large amount of analysis seems necessary to establish such proof, we may once more emphasize the usefulness of the short abbreviation $E(x)$ as was adopted here for the combined integral sine and cosine functions.

6. Some auxiliary functions. We introduce the following four functions:

$$
\begin{aligned}
& \phi_{1}(x)=\int_{0}^{x} \frac{E(x)-E(t)}{x-t} d t=-\int_{0}^{x} \log (1-t / x)\left(1-e^{-i t}\right) d t / t, \\
& \phi_{2}(x)=\int_{0}^{x} E(x-t)\left(1-e^{-i t}\right) d t / t, \\
& \phi_{3}(x)=\int_{0}^{x}\{E(x-t)-E(x)\} e^{-i t} d t / t, \\
& \phi_{4}(x)=\int_{0}^{2 x}\{\cos (x-t)-\cos x\} \log (1-t / 2 x) e^{-i t} d t / t .
\end{aligned}
$$

Between these functions the following relations exist:

$$
\begin{gathered}
2 \phi_{1}(x)+\phi_{2}(x)=2 E_{1}(x), \\
\phi_{1}(x)+\phi_{2}(x)+\phi_{3}(x)=E^{2}(x), \\
\phi_{4}(x)=e^{i x} \phi_{1}(4 x) / 2-\cos x \phi_{1}(2 x) .
\end{gathered}
$$

- C. J. Bouwkamp, Physica 9, 609-631 (1942).

- R. King and D. Middleton, Quart. Appl. Math. 3, 302-335 (1945).

${ }^{7}$ R. King and F. G. Blake, Froc. Inst. Radio Engrs. 30, 335-349 (1942). 
Formula (22) is especially noteworthy as it does not seem to be some trivial equality. A straightforward proof of it may be established by expanding both sides into powers of $x$. Evidently, $\varphi_{1}$ and $\varphi_{2}$ are integral functions; their respective power series converge for all finite values of $x$. One will get

$$
\begin{aligned}
& \phi_{1}(x)=-\sum_{n=1}^{\infty} \frac{(-i x)^{n}}{n \cdot n !}\left(1+\frac{1}{2}+\cdots+\frac{1}{n}\right), \\
& \phi_{2}(x)=2 \sum_{n=1}^{\infty} \frac{(-i x)^{n+1}}{(n+1)(n+1) !}\left(1+\frac{1}{2}+\cdots+\frac{1}{n}\right),
\end{aligned}
$$

and then (22) follows at once on account of (5).

A detailed proof of (24) only will be given as an example. Firstly,

$$
\begin{aligned}
\{\cos (x-t)-\cos x\} e^{-i t} / t & =-\frac{1}{2} e^{i x}\left(1-e^{-2 i t}\right) / t+\cos x\left(1-e^{-i t}\right) / t \\
& =\frac{d}{d t}\left\{-\frac{1}{2} e^{i x} E(2 t)+\cos x E(t)\right\} .
\end{aligned}
$$

Therefore,

$$
\phi_{4}(x)=-\int_{0}^{2 x} \log (1-t / 2 x) \frac{d}{d t}\left[\frac{1}{2} e^{i x}\{E(2 t)-E(4 x)\}-\cos x\{E(t)-E(2 x)\}\right] d t .
$$

Secondly, by partial integration,

$$
\phi_{4}(x)=\frac{1}{2} e^{i x} \int_{0}^{2 x} \frac{E(2 t)-E(4 x)}{t-2 x} d t-\cos x \int_{0}^{x} \frac{E(t)-E(2 x)}{t-2 x} d t,
$$

and, after some trivial transformation and by the use of (18), this reduces to (24).

7. Proof of formula (17). Instead of Hallén's functions $F_{n}(z)$ we take $f_{n}(z)$ $=F_{n}(z / k)$. These functions are recurrently defined by

$$
\begin{aligned}
f_{0}(z)= & \cos z, \\
f_{n+1}(z)= & \left\{f_{n}(x)-f_{n}(z)\right\} \log \left(1-z^{2} / x^{2}\right) \\
& +\int_{-x}^{x} \frac{\left\{f_{n}(x)-f_{n}(\zeta)\right\} \exp (-i|z+\zeta|)-\left\{f_{n}(x)-f_{n}(z)\right\}}{|z-\zeta|} d \zeta .
\end{aligned}
$$

Apart from $f_{0}$, only $f_{1}$ can be given explicitly in terms of known functions, viz.

$$
\begin{aligned}
f_{1}(z)= & (\cos x-\cos z) \log \left(1-z^{2} / x^{2}\right) \\
& +\frac{1}{2}\left\{e^{i z} E(2 x+2 z)+e^{-i z} E(2 x-2 z)\right\}-\cos x\{E(x+z)+E(x-z)\} .
\end{aligned}
$$

The functions $\alpha_{1}(x)$ and $\alpha_{2}(x)$ are obtained when $z=x$ is substituted in $f_{1}(z)$ and $f_{2}(z)$, respectively. Therefore the required expression for $\alpha_{2}(x)$ has to be derived from

$$
\alpha_{2}(x)=\int_{0}^{2 x}\left\{f_{1}(x)-f_{1}(x-t)\right\} \frac{e^{-i t}}{t} d t .
$$

We first write

$$
f_{1}(x)-f_{1}(x-t)=T_{1}+T_{2}+\cdots+T_{7}
$$


wherein

$$
\begin{aligned}
& T_{1}=\{\cos (x-t)-\cos x\} \log (2 t / x), \\
& T_{2}=-e^{i x} \cdot e^{i t} E(2 t) / 2, \\
& T_{3}=\cos x E(t) \\
& T_{4}=e^{i x} E(4 x)\left(1-e^{-i t}\right) / 2, \\
& T_{5}=\{\cos (x-t)-\cos x\} \log (1-t / 2 x), \\
& T_{6}=\cos x\{E(2 x-t)-E(2 x)\}, \\
& T_{7}=-e^{i x} \cdot e^{-i t}\{E(4 x-2 t)-E(4 x)\} / 2 .
\end{aligned}
$$

Let the corresponding contributions to the integral in (26) be denoted by $I_{n}$, thus

$$
I_{n}=\int_{0}^{2 x} T_{n} e^{-i t} d t / t
$$

Then one will find consecutively

$$
\begin{aligned}
& I_{1}=-\alpha_{1}(x) \log 4+e^{i x} E_{1}(4 x) / 2-\cos x E_{1}(2 x), \\
& I_{2}=-e^{-i x} E_{1}(4 x) / 2, \\
& I_{3}=\cos x\left\{E_{1}(2 x)-E^{2}(2 x) / 2\right\} \\
& I_{4}=e^{i x} E(4 x)\{E(4 x)-E(2 x)\} / 2, \\
& I_{5}=e^{i x} \phi_{1}(4 x) / 2-\cos x \phi_{1}(2 x), \\
& I_{6}=\cos x\left\{E^{2}(2 x)-\phi_{1}(2 x)-\phi_{2}(2 x)\right\}, \\
& I_{7}=e^{i x}\left\{\phi_{1}(4 x)+\phi_{2}(4 x)-E^{2}(4 x)\right\} / 2 .
\end{aligned}
$$

It is thought unnecessary to give detailed proofs of the above formulae, as the general lines are the same as in the example of the preceeding section.

Upon substituting (27a) $\cdots(33 \mathrm{a})$ in $\alpha_{2}(x)=I_{1}+I_{2}+\cdots+I_{7}$, we obtain

$$
\begin{aligned}
\alpha_{2}(x)= & \cos x E^{2}(2 x) / 2-e^{i x} E(2 x) E(4 x) / 2-\alpha_{1}(x) \log 4+i \sin x E_{1}(4 x) \\
& -\cos x\left\{2 \phi_{1}(2 x)+\phi_{2}(2 x)\right\}+e^{i x}\left\{2 \phi_{1}(4 x)+\phi_{2}(4 x)\right\} / 2 .
\end{aligned}
$$

On account of (22), the functions $\varphi_{1}$ and $\varphi_{2}$ can be eliminated. One then easily obtains the required formula (17). 
TABLE I

\begin{tabular}{|c|c|c|c|c|c|c|c|}
\hline$z$ & $\operatorname{Re} E_{1}$ & $z$ & $\operatorname{Re} E_{1}$ & $z$ & $\operatorname{Re} E_{1}$ & $z$ & $\operatorname{Re} E_{1}$ \\
\hline 0.0 & 0.000000 & 5.0 & 1.972538 & 10.0 & 3.726338 & 15.0 & 4.982566 \\
\hline 0.2 & 0.004996 & 5.2 & 2.066256 & 10.2 & 3.784619 & 15.2 & 5.025625 \\
\hline 0.4 & 0.019933 & 5.4 & $2.157 \quad 214$ & 10.4 & 3.842420 & 15.4 & 5.068443 \\
\hline 0.6 & 0.044664 & 5.6 & 2.245360 & 10.6 & 3.899692 & 15.6 & 5.111035 \\
\hline 0.8 & 0.078943 & 5.8 & 2.330699 & 10.8 & 3.956381 & 15.8 & 5.153410 \\
\hline 1.0 & $0.122 \quad 434$ & 6.0 & 2.413282 & 11.0 & 4.012436 & 16.0 & 5.195569 \\
\hline 1.2 & $0.174 \quad 714$ & 6.2 & 2.493205 & 11.2 & 4.067808 & 16.2 & 5.237508 \\
\hline 1.4 & 0.235281 & 6.4 & 2.570598 & 11.4 & $4.122 \quad 452$ & 16.4 & 5.279217 \\
\hline 1.6 & 0.303564 & 6.6 & 2.645618 & 11.6 & 4.176332 & 16.6 & 5.320681 \\
\hline 1.8 & 0.378933 & 6.8 & $2.718 \quad 446$ & 11.8 & 4.229418 & 16.8 & 5.361884 \\
\hline 2.0 & 0.460706 & 7.0 & 2.789276 & 12.0 & 4.281693 & 17.0 & 5.402804 \\
\hline 2.2 & $\begin{array}{lll}0.548 & 165\end{array}$ & 7.2 & $2.85831 \mathrm{C}$ & 12.2 & $4.333 \quad 147$ & 17.2 & 5.443422 \\
\hline 2.4 & 0.640563 & 7.4 & 2.925751 & 12.4 & $4.383 \quad 783$ & 17.4 & 5.483715 \\
\hline 2.6 & $0.737 \quad 142$ & 7.6 & 2.991799 & 12.6 & $4.433 \quad 614$ & 17.6 & 5.523663 \\
\hline 2.8 & $0.837 \quad 139$ & 7.8 & 3.056642 & 12.8 & 4.482661 & 17.8 & $\begin{array}{lll}5.563 & 247\end{array}$ \\
\hline 3.0 & 0.939800 & 8.0 & 3.120458 & 13.0 & $4.530 \quad 954$ & 18.0 & 5.602453 \\
\hline 3.2 & $1.044 \quad 392$ & 8.2 & 3.183403 & 13.2 & 4.578533 & 18.2 & $\begin{array}{lll}5.641 & 267\end{array}$ \\
\hline 3.4 & 1.150210 & 8.4 & 3.245618 & 13.4 & $4.625 \quad 441$ & 18.4 & 5.679683 \\
\hline 3.6 & 1.256591 & 8.6 & $3.307 \quad 218$ & 13.6 & $4.671 \quad 727$ & 18.6 & 5.717694 \\
\hline 3.8 & 1.362916 & 8.8 & 3.368298 & 13.8 & 4.717442 & 18.8 & $5.755 \quad 304$ \\
\hline 4.0 & 1.468623 & 9.0 & 3.428929 & 14.0 & $4.762 \quad 639$ & 19.0 & 5.792515 \\
\hline 4.2 & 1.573207 & 9.2 & 3.489159 & 14.2 & 4.807370 & 19.2 & $\begin{array}{lll}5.829 & 339\end{array}$ \\
\hline 4.4 & 1.676231 & 9.4 & 3.549016 & 14.4 & $\begin{array}{lll}4.851 & 684\end{array}$ & 19.4 & 5.865788 \\
\hline 4.6 & 1.777320 & 9.6 & $3.608 \quad 507$ & 14.6 & $4.895 \quad 628$ & 19.6 & 5.901879 \\
\hline 4.8 & 1.876168 & 9.8 & 3.667622 & 14.8 & $4.939 \quad 244$ & 19.8 & $5.937 \quad 632$ \\
\hline 5.0 & 1.972538 & 10.0 & 3.726338 & 15.0 & $4.982 \quad 566$ & 20.0 & 5.973068 \\
\hline
\end{tabular}

TABLE II

\begin{tabular}{|c|c|c|c|c|c|c|c|}
\hline$z$ & $\operatorname{Im} E_{1}$ & $z$ & $\operatorname{Im} E_{1}$ & $z$ & $\operatorname{Im} E_{1}$ & $z$ & $\operatorname{Im} E_{1}$ \\
\hline 0.0 & 0.000000 & 5.0 & 3.467907 & 10.0 & $4.526 \quad 334$ & 15.0 & 5.157090 \\
\hline 0.2 & 0.199852 & 5.2 & 3.527976 & 10.2 & 4.559056 & 15.2 & 5.178576 \\
\hline 0.4 & $0.398 \quad 818$ & 5.4 & 3.584497 & 10.4 & $4.590 \quad 878$ & 15.4 & 5.199864 \\
\hline 0.6 & 0.596026 & 5.6 & 3.637923 & 10.6 & 4.621791 & 15.6 & 5.220929 \\
\hline 0.8 & $0.790 \quad 627$ & 5.8 & $3.688 \quad 683$ & 10.8 & 4.651802 & 15.8 & $5.241 \quad 742$ \\
\hline 1.0 & 0.981811 & 6.0 & $3.737 \quad 180$ & 11.0 & 4.680931 & 16.0 & $5.262 \quad 280$ \\
\hline 1.2 & 1.168815 & 6.2 & $3.783 \quad 779$ & 11.2 & $4.709 \quad 208$ & 16.2 & $5.282 \quad 518$ \\
\hline 1.4 & 1.350936 & 6.4 & 3.828813 & 11.4 & 4.736676 & 16.4 & $5.302 \quad 436$ \\
\hline 1.6 & 1.527537 & 6.6 & 3.872571 & 11.6 & 4.763386 & 16.6 & $5.322 \quad 019$ \\
\hline 1.8 & 1.698057 & 6.8 & $3.915 \quad 302$ & 11.8 & $4.789 \quad 398$ & 16.8 & $5.341 \quad 255$ \\
\hline 2.0 & 1.862017 & 7.0 & 3.957213 & 12.0 & $4.814 \quad 776$ & 17.0 & 5.360140 \\
\hline 2.2 & 2.019023 & 7.2 & 3.998470 & 12.2 & 4.839587 & 17.2 & $5.378 \quad 671$ \\
\hline 2.4 & 2.168772 & 7.4 & $\begin{array}{lll}4.039 & 198\end{array}$ & 12.4 & 4.863898 & 17.4 & 5.396855 \\
\hline 2.6 & 2.311048 & 7.6 & 4.079485 & 12.6 & $4.887 \quad 779$ & 17.6 & $5.414 \quad 701$ \\
\hline 2.8 & $2.445 \quad 729$ & 7.8 & 4.119385 & 12.8 & $\begin{array}{lll}4.911291\end{array}$ & 17.8 & $5.432 \quad 223$ \\
\hline 3.0 & $2.572 \quad 779$ & 8.0 & 4.158921 & 13.0 & $4.934 \quad 494$ & 18.0 & $5.449 \quad 442$ \\
\hline 3.2 & 2.692246 & 8.2 & $4.198 \quad 089$ & 13.2 & 4.957441 & 18.2 & $5.466 \quad 378$ \\
\hline 3.4 & $2.804 \quad 259$ & 8.4 & 4.236865 & 13.4 & $\begin{array}{lll}4.980 & 178\end{array}$ & 18.4 & 5.483057 \\
\hline 3.6 & 2.909021 & 8.6 & 4.275206 & 13.6 & 5.002741 & 18.6 & $5.499 \quad 504$ \\
\hline 3.8 & 3.006798 & 8.8 & 4.313058 & 13.8 & $\begin{array}{lll}5.025 & 158\end{array}$ & 18.8 & $5.515 \quad 747$ \\
\hline 4.0 & 3.097916 & 9.0 & 4.350357 & 14.0 & 5.047448 & 19.0 & $\begin{array}{lll}5.531 & 813\end{array}$ \\
\hline 4.2 & 3.182750 & 9.2 & 4.387037 & 14.2 & 5.069623 & 19.2 & $5.547 \quad 727$ \\
\hline 4.4 & 3.261713 & 9.4 & 4.423033 & 14.4 & 5.091683 & 19.4 & $5.563 \quad 513$ \\
\hline 4.6 & $3.335 \quad 250$ & 9.6 & $4.458 \quad 283$ & 14.6 & 5.113624 & 19.6 & $\begin{array}{lll}5.579 & 192\end{array}$ \\
\hline 4.8 & 3.403823 & 9.8 & $4.492 \quad 731$ & 14.8 & $5.135 \quad 432$ & 19.8 & $5.594 \quad 782$ \\
\hline 5.0 & 3.467907 & 10.0 & $4.526 \quad 334$ & 15.0 & 5.157090 & 20.0 & 5.610298 \\
\hline
\end{tabular}


TABle III

\begin{tabular}{|c|c|c|c|c|c|c|}
\hline$x$ & & $\alpha_{1}^{I}$ & $\alpha_{1}^{\text {II }}$ & $x$ & $\alpha_{1}^{I}$ & $\alpha_{1}^{\text {II }}$ \\
\hline 0.0 & & 0.0000 & 0.0000 & 2.5 & 0.2361 & 1.4528 \\
\hline 0.1 & & -0.0100 & 0.0007 & 2.6 & 0.3550 & 1.3695 \\
\hline 0.2 & & -0.0393 & 0.0053 & 2.7 & 0.4686 & 1.2690 \\
\hline 0.3 & & -0.0865 & 0.0175 & 2.8 & 0.5756 & 1.1534 \\
\hline 0.4 & & -0.1490 & 0.0407 & 2.9 & 0.6749 & 1.0247 \\
\hline 0.5 & & -0.2235 & 0.0773 & 3.0 & 0.7661 & 0.8851 \\
\hline 0.6 & & -0.3061 & 0.1292 & 3.1 & 0.8487 & 0.7362 \\
\hline 0.7 & & -0.3924 & 0.1973 & 3.2 & 0.9225 & 0.5800 \\
\hline 0.8 & & -0.4780 & 0.2815 & 3.3 & 0.9875 & 0.4180 \\
\hline 0.9 & & -0.5583 & 0.3807 & 3.4 & 1.0436 & 0.2514 \\
\hline 1.0 & & -0.6291 & 0.4930 & 3.5 & 1.0905 & 0.0816 \\
\hline 1.1 & & -0.6866 & 0.6156 & 3.6 & 1.1280 & -0.0904 \\
\hline 1.2 & & -0.7278 & 0.7451 & 3.7 & 1.1556 & -0.2634 \\
\hline 1.3 & & -0.7503 & 0.8777 & 3.8 & 1.1730 & -0.4363 \\
\hline 1.4 & & -0.7528 & 1.0091 & 3.9 & 1.1795 & -0.6080 \\
\hline 1.5 & & -0.7345 & 1.1351 & 4.0 & 1.1743 & -0.7771 \\
\hline 1.6 & & -0.6957 & 1.2517 & 4.1 & 1.1569 & -0.9422 \\
\hline 1.7 & & -0.6376 & $1 . .5550$ & 4.2 & 1.1265 & -1.1016 \\
\hline 1.8 & & -0.5618 & 1.4419 & 4.3 & 1.0828 & -1.2535 \\
\hline 1.9 & & -0.4708 & 1.5097 & 4.4 & 1.0253 & -1.3960 \\
\hline 2.0 & & -0.3672 & 1.5563 & 4.5 & 0.9542 & -1.5271 \\
\hline 2.1 & & -0.2540 & 1.5805 & 4.6 & 0.8695 & -1.6446 \\
\hline 2.2 & - & -0.1343 & 1.5819 & 4.7 & 0.7720 & -1.7468 \\
\hline 2.3 & & -0.0109 & 1.5604 & 4.8 & $0.66^{\prime} 25$ & -1.8315 \\
\hline 2.4 & - & 0.1134 & 1.5170 & 4.9 & 0.5423 & -1.8973 \\
\hline 2.5 & & 0.2361 & 1.4528 & 5.0 & 0.4130 & -1.9426 \\
\hline
\end{tabular}

TABLE IV

\begin{tabular}{|c|c|c|c|c|c|}
\hline$x$ & $\alpha_{2}^{I}$ & $\alpha_{2}^{I I}$ & $x$ & $\alpha_{2}^{I}$ & $\alpha_{2}^{\text {II }}$ \\
\hline 0.0 & 0.0000 & 0.0000 & 2.5 & -2.5787 & 3.5091 \\
\hline 0.1 & -0.0359 & 0.0022 & 2.6 & -2.2678 & 3.4640 \\
\hline 0.2 & -0.1415 & 0.0171 & 2.7 & -1.9045 & 3.3755 \\
\hline 0.3 & -0.3099 & 0.0563 & 2.8 & -1.4931 & 3.2385 \\
\hline 0.4 & -0.5311 & 0.1284 & 2.9 & -1.0397 & 3.0487 \\
\hline 0.5 & -0.7919 & 0.2392 & 3.0 & -0.5524 & 2.8034 \\
\hline 0.6 & -1.0784 & 0.3901 & 3.1 & -0.0402 & 2.5018 \\
\hline 0.7 & -1.3762 & 0.5790 & 3.2 & 0.4869 & 2.1447 \\
\hline 0.8 & -1.6723 & 0.8004 & 3.3 & 1.0187 & 1.7349 \\
\hline 0.9 & -1.9560 & 1.0462 & 3.4 & 1.5450 & 1.2770 \\
\hline 1.0 & -2.2191 & 1.3070 & 3.5 & 2.0560 & 0.7771 \\
\hline 1.1 & -2.4564 & 1.5727 & 3.6 & 2.5430 & 0.2427 \\
\hline 1.2 & -2.6654 & 1.8344 & 3.7 & 2.9979 & -0.3177 \\
\hline 1.3 & -2.8455 & 2.0844 & 3.8 & 3.4141 & -0.8948 \\
\hline 1.4 & -2.9977 & 2.3171 & 3.9 & 3.7862 & -1.4792 \\
\hline 1.5 & -3.1232 & 2.5291 & 4.0 & 4.1100 & -2.0611 \\
\hline 1.6 & -3.2229 & 2.7192 & 4.1 & 4.3828 & -2.6312 \\
\hline 1.7 & -3.2969 & 2.8875 & 4.2 & 4.6030 & -3.1808 \\
\hline 1.8 & -3.3437 & 3.0355 & 4.3 & 4.7700 & -3.7021 \\
\hline 1.9 & -3.3604 & 3.1644 & 4.4 & 4.8840 & -4.1880 \\
\hline 2.0 & -3.3431 & 3.2752 & 4.5 & 4.9459 & -4.6330 \\
\hline 2.1 & -3.2867 & 3.3680 & 4.6 & 4.9568 & -5.0323 \\
\hline 2.2 & -3.1862 & 3.4413 & 4.7 & 4.9180 & -5.3825 \\
\hline 2.3 & -3.0369 & 3.4923 & 4.8 & 4.8308 & -5.6811 \\
\hline 2.4 & -2.8350 & 3.5166 & 4.9 & 4.6959 & -5.9264 \\
\hline 2.5 & -2.5787 & 3.5091 & 5.0 & 4.5142 & -6.1172 \\
\hline
\end{tabular}

